\title{
Involvement of Drp1 in hypoxia-induced migration of human glioblastoma $\mathrm{U} 251$ cells
}

\author{
YU-YING WAN $^{1,2^{*}}$, JIAN-FENG ZHANG ${ }^{1 *}$, ZHANG-JIAN YANG $^{1 *}$, LI-PING JIANG ${ }^{3}$, YONG-FANG WEI ${ }^{1}$, \\ QI-NAN LAI ${ }^{1}$, JIAN-BIN WANG ${ }^{1}$, HONG-BO XIN $^{1}$ and XIAO-JIAN HAN ${ }^{1}$ \\ ${ }^{1}$ Institute of Translational Medicine, Nanchang University, Nanchang, Jiangxi; ${ }^{2}$ Department of Hospital Infection, \\ The Second Affiliated Hospital of Nanchang University, Nanchang, Jiangxi; ${ }^{3}$ Department of Pharmacology, \\ Nanchang University School of Pharmaceutical Science, Nanchang, Jiangxi, P.R. China
}

Received March 23, 2014; Accepted May 21, 2014

DOI: $10.3892 /$ or.2014.3235

\begin{abstract}
Glioblastoma is one of the most aggressive brain tumors with high morbidity and mortality. Hypoxia is often the common characteristic of tumor microenvironment, and hypoxia-inducible factor- $1 \alpha(\mathrm{HIF}-1 \alpha)$ is an essential factor regulating the migratory activity of cancer cells including glioblastoma. Recently, mitochondrial dynamics was found to be involved in the aggression of cancer cells. However, whether dynamin-related protein 1 (Drp1) contributes to the migration of human glioblastoma cells under hypoxia remains unknown. In the present study, hypoxia was found to upregulate the transcription and expression of Drp1, and stimulated mitochondrial fission in glioblastoma U251 cells. Inhibition of HIF-1 $\alpha$ with echinomycin blocked hypoxia-induced expression of Drp1. Notably, Drp1 inhibitor Mdivi-1 efficiently attenuated hypoxia-induced mitochondrial fission and migration of U251 cells. In addition, three U251 stable cell lines expressing GFP, GFP-Drp1 and dominant negative GFP-Drp1-K38A were established to examine the direct role of Drp1 in hypoxiainduced migration. MTT assay showed that there was no significant difference in proliferation of three cell lines. Compared with the GFP cell line, exogenously expressed GFP-Drp1-K38A inhibited hypoxia-induced migration of U251 cells, while stable expression of GFP-Drp1 enhanced the migration of U251 cells under hypoxia. Therefore, this study indicates the involvement of Drp1 in hypoxia-induced migration of human glioblastoma U251 cells, and suggests Drp1 to be a potential therapeutic target to suppress the aggression of glioblastoma in the future.
\end{abstract}

Correspondence to: Dr Xiao-Jian Han, Institute of Translational Medicine, Nanchang University, 1299 Xuefu Road, Honggu District, Nanchang, Jiangxi 330031, P.R. China

E-mail: hanxiaojian@hotmail.com

${ }^{*}$ Contributed equally

Key words: Drp1, hypoxia, migration, glioblastoma, mitochondria

\section{Introduction}

Glioblastoma is one of the most malignant and aggressive cerebral gliomas. The strong aggression always leads to diffuse invasion of glioblastoma cells into adjacent normal brain tissue and migration to a considerable distance from the primary tumor area $(1,2)$. Despite the advances in surgery and radiotherapy for glioblastoma, it is still difficult to remove all tumor tissues without severe damage to the brain, and avoid vulnerability of healthy brain tissue to radiotherapy, resulting in unsatisfactory therapeutic efficacy and poor prognosis of glioblastoma (3). Thus, it is necessary to further investigate the molecular mechanism regulating the aggression of glioblastoma cells. Increasing evidence suggests the microenvironment in solid tumors plays a vital role in migration and invasion. Hypoxia is the common characteristic of tumor microenvironment, and also the major stimulator of migration and invasion in tumors $(4,5)$. It is well documented that hypoxia modifies cellular activities via stabilizing hypoxia-inducible factor- $1 \alpha(\mathrm{HIF}-1 \alpha)$. Under normoxia, $\mathrm{O}_{2}$-dependent enzymatic hydroxylation at P402 and/or P564 leads to ubiquitination and proteasomal degradation of HIF-1 $\alpha$. However, under hypoxia in tumor microenvironment, HIF-1 $\alpha$ remains unhydroxylated and stable against proteasomal degradation (6,7). As a transcription factor, HIF-1 $\alpha$ promotes the adaptation of tumor cells to hypoxia through upregulating the expression of genes related to angiogenesis, glycolysis and cellular mobility, such as VEGF, GLUT1 and MMPs $(4,8,9)$. In glioblastoma, HIF-1 $\alpha$ is often detected predominantly in hypoxic regions and in tumor cells infiltrating the normal brain tissue (10). This suggests the possible role of HIF-1 $\alpha$ in the aggressiveness of glioblastoma.

Mitochondria play an essential function in cells through the production of energy and the ability to regulate intracellular $\mathrm{Ca}^{2+}$. As such, they are involved in a variety of cellular processes, including survival, proliferation and apoptosis (11-13). These functions are crucial for cancer cells, since cancer is characterized as a disease of inappropriate cell proliferation and dysregulated cell cycle control. On the other hand, mitochondria are also dynamic organelles and move through the cell with frequent fission and fusion events. The dynamic balance of fission and fusion events is important to 
maintain the normal shape, structure and function of mitochondria (14). It has been demonstrated that the dynamic mitochondrial morphology corresponds to the metabolic status of cells, and unbalanced mitochondrial fission and fusion events potentially contribute to tumorigenesis (15). In addition, some highly conserved dynamin-related GTPases are identified as the mediator of mitochondrial dynamics. Dynamin-related protein 1 (Drp1) is involved in the process of mitochondrial fission, while mitofusins and OPA1 are required for mitochondrial fusion in mammalian cells $(14,15)$. Recent studies demonstrated the involvement of Drp1 in the development of lung and breast cancer and neuroblastoma (16-18). Inhibition of Drpl efficiently reduced cancer cell growth and enhanced spontaneous apoptosis in several types of cancer, including lung, breast, colon and cervical cancer (16,19-21). Thus, Drpl is considered as a potential therapeutic target of cancer in the future. In addition, a recent study demonstrated the role of Drp1 in the migration and invasion of breast cancer cells. The high expression of Drp1 leads to more fragmented mitochondria and redistribution of mitochondria to lamellipodial regions. Drp1-dependent mitochondrial fission promotes lamellipodia formation at the leading edge of breast cancer cells, which is critical for their migration and invasion (17). However, whether Drp1 contributes to the migration of human glioblastoma cells under hypoxia remains unknown.

In the present study, we first examined the effect of hypoxia on the transcription and expression of Drp1 in glioblastoma U251 cells. It was found that hypoxia upregulated the transcription and expression of Drp1, and consequently increased mitochondrial fission. The migration of U251 cells was evaluated by scratch and Transwell assay. Consistent with previous studies (5), $\mathrm{CoCl}_{2}$ and hypoxic incubation promoted the migration of U251 cells. To test the involvement of Drp1 in hypoxia-induced migration, the effect of Mdivi-1, a Drp1 inhibitor, on the migration of U251 cells was examined. Results showed that Mdivi-1 efficiently attenuated hypoxia-induced migration of U251 cells. To demonstrate the direct role of Drp1 in hypoxia-induced migration of U251, we established three U251 stable cell lines expressing GFP, GFP-Drp1 and dominant negative GFP-Drp1-K38A. As expected, exogenously expressed GFP-Drp1 enhanced hypoxia-induced migration, while expression of dominant negative GFP-Drp1-K38A inhibited the migration of U251. Collectively, our data demonstrate for the first time that mitochondrial fission protein Drp1 is involved in hypoxia-induced migration of human glioblastoma U251 cells. Inhibition of Drp1-dependent mitochondrial fission may be a potential strategy for prevention and therapy of glioblastoma in the future.

\section{Materials and methods}

Materials. Fetal bovine serum (FBS), Dulbecco's modified Eagle's medium (DMEM), trypsin and the solution of penicillin-streptomycin $(\mathrm{P} / \mathrm{S})$ were purchased from Gibco. $\mathrm{CoCl}_{2}, \mathrm{G} 418$ and Drp1 inhibitor Mdivi-1 were purchased from Sigma. Transfection kits including Lipofectamine 2000 and Opti-MEM were from Invitrogen. Dimethyl sulfoxide (DMSO) was purchased from Solarbio (Beijing, China). Primers for qRT-PCR were synthesized by Sangon Biotech Co. (Shanghai, China). PrimeScript ${ }^{\mathrm{TM}}$ RT reagent kit with gDNA Eraser and
SYBR® Premix Ex Taq $^{\mathrm{TM}}$ II were from Takara. The RNA simple total RNA kit was from Tiangen (Beijing, China). Amersham ECL prime western blotting detection reagents were from GE Healthcare.

Cell culture, scratch and Transwell assay. Human U251 glioblastoma cell line was obtained from the American Type Culture Collection (ATCC). U251 cells were grown in a DMEM medium supplemented with $10 \%$ FBS and $100 \mu \mathrm{g} / \mathrm{ml}$ $\mathrm{P} / \mathrm{S}$ in a humidified incubator at $37^{\circ} \mathrm{C}$ with an atmosphere containing $5 \% \mathrm{CO}_{2}$. For hypoxia treatment, the cells were incubated in hypoxia $\left(1 \% \mathrm{O}_{2}, 5 \% \mathrm{CO}_{2}\right.$ and $\left.94 \% \mathrm{~N}_{2}\right)$ or treated with $100 \mu \mathrm{M} \mathrm{CoCl}_{2}$. The scratch and Transwell assays were carried out as previously described (22). In the scratch assay, the cells firstly were seeded on $35 \mathrm{~mm}$ dishes and grown in growth medium. Treatment with $100 \mu \mathrm{M} \mathrm{CoCl}_{2}$ was used to mimic hypoxia in U251 cells. Briefly, a scratch was made in monolayer of cells with a $200-\mu 1$ pipette tip. Then, the cells were washed twice with PBS to remove the suspended cells and incubated in DMEM without supplement of FBS and P/S, or DMEM containing $5 \mu \mathrm{M}$ Mdivi-1 or DMSO. The migration status was photographed by bright-field microscopy at different time points after scratch. Transwell assay was performed with Transwell chamber (Corning). In brief, $1.2 \times 10^{4}$ cells were seeded into the upper chamber in $200 \mu \mathrm{l}$ of serum-free medium, while the bottom of the chamber was incubated with $500 \mu \mathrm{l}$ of complete medium containing $10 \% \mathrm{FBS}$ and $1 \% \mathrm{P} / \mathrm{S}$. After $6 \mathrm{~h}$ of migration, the cells on the top surface of the insert were gently removed with a cotton swab. The migrated cells on the lower surface were fixed with $4 \%$ paraformaldehyde and stained with crystal violet for $30 \mathrm{~min}$, and counted under a microscope. All assays were independently repeated at least in triplicate.

Quantitative real-time PCR. Total RNA was extracted from cells cultured in DMEM or DMEM containing $100 \mu \mathrm{M} \mathrm{CoCl}_{2}$ using an RNAsimple Total RNA kit (Tiangen). Total RNA was reverse-transcribed using PrimeScript ${ }^{\mathrm{TM}}$ RT reagent kit with gDNA Eraser. Quantitative RT-PCR was performed using SYBR $^{\circledR}$ Premix Ex Taq $^{\mathrm{TM}}$ II and a 7500 Real-Time PCR System (Applied Biosystems). Relative quantification of gene expression was calculated using the formula: $2^{-\Delta \Delta \mathrm{Ct}}, \Delta \Delta \mathrm{Ct}=$ $\left(C t_{\text {target gene }}-C t_{\mathrm{GAPDH}}\right)_{\text {hypoxia }}-\left(C t_{\text {target gene }}-C t_{\mathrm{GAPDH}}\right)_{\text {normoxia }}$. The qRT-PCR of Drp1 was performed using a sense primer, 5'-TG AAGGATGTCATGTCGGACC-3' and an antisense primer, 5'-GTTGAGGACGTTGACTTGGCT-3'. The primers for GAPDH were: 5'-CAGGGCTGCTTTTAACTCTGGT-3' (sense), and 5'-GATTTTGGAGGGATCTCGCT-3' (antisense). Three independent experiments for each condition were carried out.

Western blot analysis. The U251 cells or the three U251 stable cell lines expressing GFP, GFP-Drp1 or GFP-Drp1-K38A after hypoxia treatment were harvested and lysed. The cell lysates were subjected to $8 \%$ SDS-PAGE gel electrophoresis. After electrophoresis, the proteins were transferred onto polyvinylidene difluoride (PVDF) membrane (Millipore, USA). The membrane was blocked with $5 \%$ skim milk in TBST buffer for $1 \mathrm{~h}$ at room temperature. Then, the PVDF membranes were immunoblotted with mouse anti-Drp1 antibody 
(BD Biosciences) at a dilution of 1:1,000. After three washes with TBST, the membranes were further incubated with an HRP-conjugated relative secondary antibody (at a dilution of 1:2,000) for $2 \mathrm{~h}$ at room temperature. Chemiluminescence assay were performed with Amersham ECL prime western blotting detection reagents, and the immunobloting signal was detected using Molecular Imager ${ }^{\circledR}$ ChemiDoc $^{\mathrm{T}} \mathrm{XRS}^{+}$System (Bio-Rad).

Mitochondrial imaging. As previously described (32), pDsRed2-Mito was transfected into U251 cells to label mitochondria with Lipofectamine 2000. Twenty-four hours after transfection, mitochondrial morphology was observed with an inverted fluorescence microscope (Axiovert 200; Carl Zeiss Inc.) with excitation at $545 \mathrm{~nm}$. After $6 \mathrm{~h}$ hypoxia treatment, U251 cells were fixed with $4 \%$ PFA, and mitochondrial morphology was detected again under fluorescence microscope after hypoxic treatment for $12 \mathrm{~h}$. To examine the role of Drp1 on hypoxia-induced mitochondrial morphology, cells were pretreated with $5 \mu \mathrm{M}$ Mdivi-1 $1 \mathrm{~h}$ prior to hypoxia.

Establishment of U251 stable cell lines expressing GFP, GFP-DRP1 and GFP-Drpl-K38A. Firstly, the minimum lethal concentration of G418 was determined as previously described (22). Briefly, U251 cells were seeded in 12-well plates at a density of $5 \times 10^{4}$. The cells were cultured in growth medium containing $0-1,100 \mu \mathrm{g} / \mu \mathrm{l}$ of G418 for 12 days. Growth medium containing different concentrations of G418 was exchanged every 3-4 days. The survival state of cells was monitored every $24 \mathrm{~h}$ under a bright-field microscope. The minimum concentration of G418 that induced complete cell death in 12-well plates was the minimum lethal concentration of G418 to U251 cells. GFP, GFP-Drp1 and GFP-Drp1-K38A plasmids were transfected into U251 cells with Lipofectamine 2000 according to the manufacturer's instructions. Forty-eight hours after transfection, the cells were selected with a higher concentration than minimum lethal concentration of G418. The selected colonies with GFP fluorescence were picked up and passaged. The cell lines stably expressing GFP, GFP-Drp1 or GFP-Drp1-K38A were further confirmed by fluorescence microscopy and western blotting.

MTT assay. The 3-(4,5-dimethylthiazol-2-yl)-2,5-diphenyltetrazolium bromide (MTT) assay was applied to assess the proliferation of three U251 cell lines according to the manufacturer's instructions. Approximately, 5x103 cells were seeded in 96-well tissue culture plates and cultured in growth medium. The proliferation of U251 cells was detected after the cells cultured at $37^{\circ} \mathrm{C}, 5 \% \mathrm{CO}_{2}$ for $12,24,48$ and $72 \mathrm{~h}$. Twenty microliters of the MTT solution was added to each well (5 $\mathrm{mg} / \mathrm{ml}, 0.5 \% \mathrm{MTT})$ and the cells continued to culture for $4 \mathrm{~h}$. After the incubation, the supernatant was discarded. DMSO $(100 \mu \mathrm{l})$ was added to each well, and the culture plate was shaken at low-speed for 10 min until the crystal dissolved completely. The color intensity was measured spectrophotometrically using a microplate reader (Thermo Multiskan ${ }^{\circledR}$ FC) at $570 \mathrm{~nm}$. All assays were performed at least three times.

Statistical analysis. The quantitative data are shown as the means \pm SD. Data were analyzed using either Student's t-test
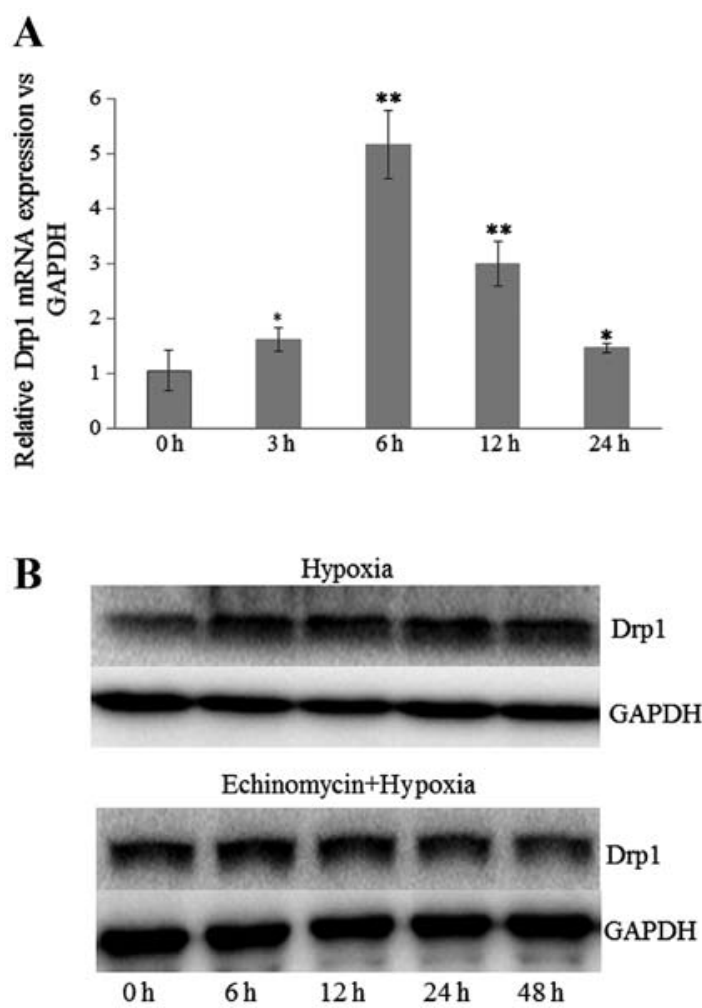

Figure 1. Effect of $\mathrm{CoCl}_{2}$ and hypoxia treatment on the transcription and expression of Drp1 in U251 cells. (A) The mRNA level of Drp1 in U251 cells after $\mathrm{CoCl}_{2}$ treatment. Quantitative RT-PCR was applied to detect the gene transcription of Drp1 in U251 at 3-24 h after $\mathrm{CoCl}_{2}$ treatment. The transcription of GAPDH was used as an endogenous reference. The mRNA level of Drp1 after $\mathrm{CoCl}_{2}$ treatment was normalized to that of PBS treatment (control). Three independent experiments for each condition were carried out. ${ }^{*} \mathrm{p}<0.01,{ }^{* *} \mathrm{p}<0.001$ (compared with control). (B) The expression of Drp1 in U251 cells after hypoxia treatment. Cells were pretreated with $2 \mu \mathrm{g} / \mathrm{ml}$ of echinomycin or vehicle for $30 \mathrm{~min}$. The lysate of U251 cells was prepared at indicated time points after hypoxia treatment. Expression of Drpl was detected using immunoblot assay after $8 \%$ SDS-PAGE. GAPDH was used as endogenous reference. Drp1, dynamin-related protein 1.

to compare two conditions or ANOVA followed by planned comparisons of multiple conditions, and $\mathrm{p}<0.05$ was considered to indicate a statistically significant difference.

\section{Results}

Hypoxia upregulates the transcription and expression of Drpl and stimulates mitochondrial fission in glioblastoma U251 cells. A recent study found Drp1-dependent mitochondrial fission could enhance the migration and invasion of breast cancer cells through modifying lamellipodial formation (17). On the other hand, $\mathrm{CoCl}_{2}$ is a common chemical HIF-1 $\alpha$ activator $(23,24,36)$, and HIF-1 $\alpha$ promotes the adaption of cancer cells to hypoxia through upregulating some genes related to cellular mobility, angiogenesis and glycolysis (4,5). In the present research, we investigated whether Drp1 is also involved in hypoxia-induced migration of human glioblastoma U251 cells. To test the hypothesis, the mRNA expression of Drp1 in $\mathrm{U} 251$ cells after $\mathrm{CoCl}_{2}$ treatment was examined by quantitative RT-PCR. As shown in Fig. 1A, the mRNA expression of Drp1 was significantly increased from $3 \mathrm{~h}$ post-treatment. The transcription level of Drp1 was increased $\sim 5$-fold at $6 \mathrm{~h}$ 

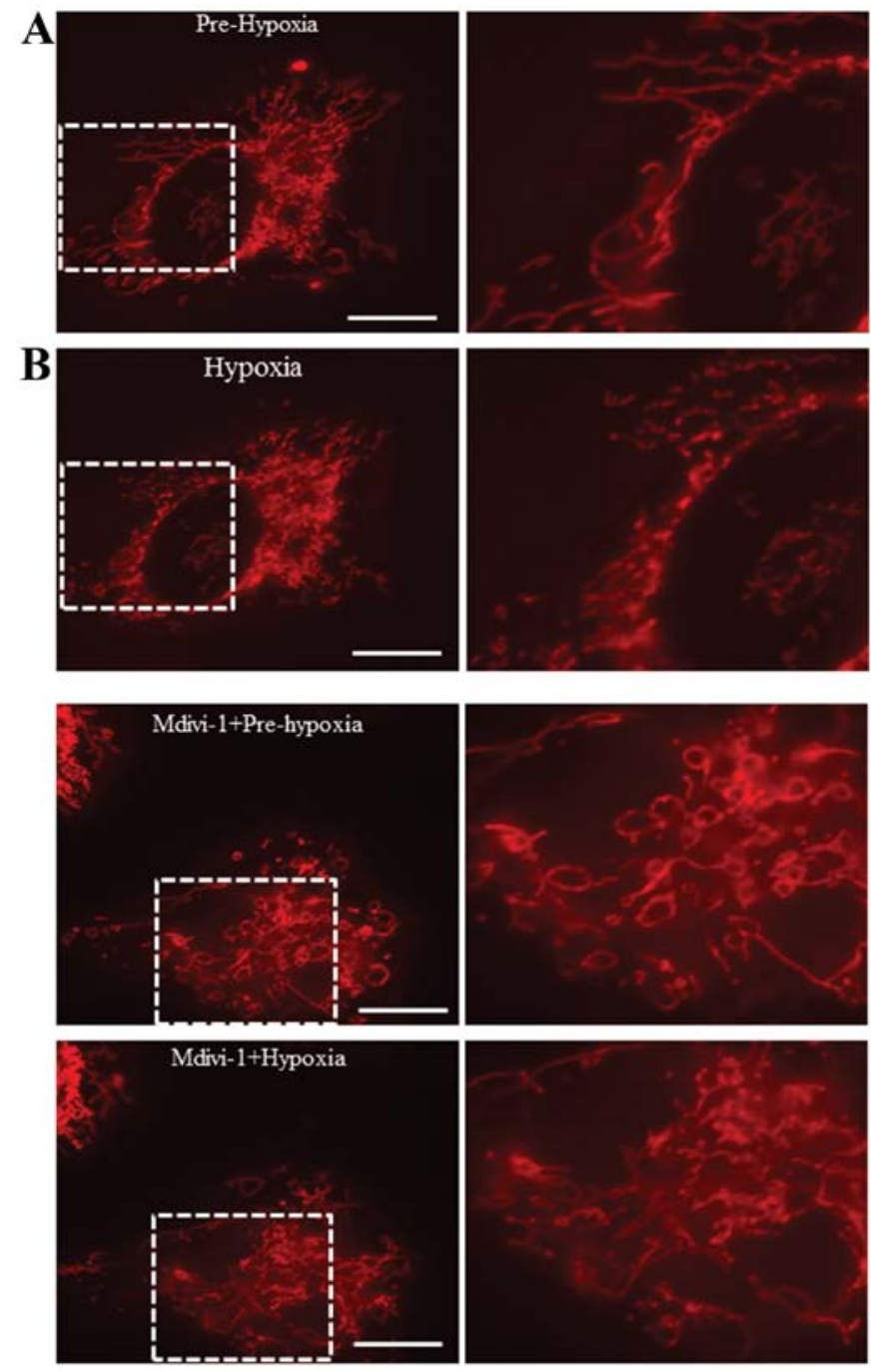

Figure 2. Hypoxia stimulates Drp1-dependent mitochondrial fission in U25 cells. pDsRed2-Mito was transfected into U251 cells to label mitochondria. Cells were pretreated with $5 \mu \mathrm{M}$ of Mdivi-1 (B) or vehicle (A), and incubated in $1 \% \mathrm{O}_{2}$ for $8 \mathrm{~h}$. Mitochondrial morphology was detected pre- and post-hypoxia. Right panels show mitochondria in the indicated regions with higher magnification. Scale bar, $10 \mu \mathrm{m}$. Drp1, dynamin-related protein 1 .

post-treatment with $\mathrm{CoCl}_{2}$. In addition, the protein level of Drp1 after hypoxia treatment $\left(1 \% \mathrm{O}_{2}, 5 \% \mathrm{CO}_{2}\right.$ and $\left.94 \% \mathrm{~N}_{2}\right)$ was also examined by western blot assay. Consistent with the results of mRNA expression, the expression of Drp1 in U251 cells was also upregulated by hypoxia treatment. Notably, pretreatment with inhibitor of HIF-1 $\alpha$, echinomycin, blocked hypoxia-induced expression of Drp1 (Fig. 1B). Drp1 is a large GTPase mediating mitochondrial fission. Thus, mitochondrial morphology was examined to evaluate the function of high expressed Drp1 under hypoxia. As shown in Fig. 2, mitochondrial fission was increased after hypoxia treatment, while pretreatment with Drp1 inhibitor Mdivi-1 efficiently attenuated hypoxia-induced mitochondrial fission. These results suggest that hypoxia may upregulate the transcription and expression of Drp1 through activation of HIF-1 $\alpha$ and subsequently enhance mitochondrial fission.

Effect of Drpl inhibitor Mdivi-1 on hypoxia-induced migration of U251 cells. Hypoxia is an important factor to stimulate

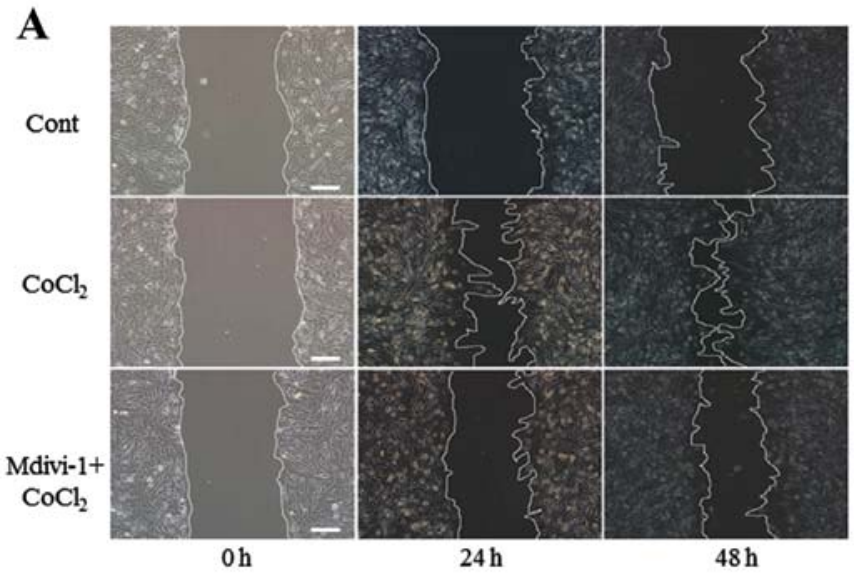

B
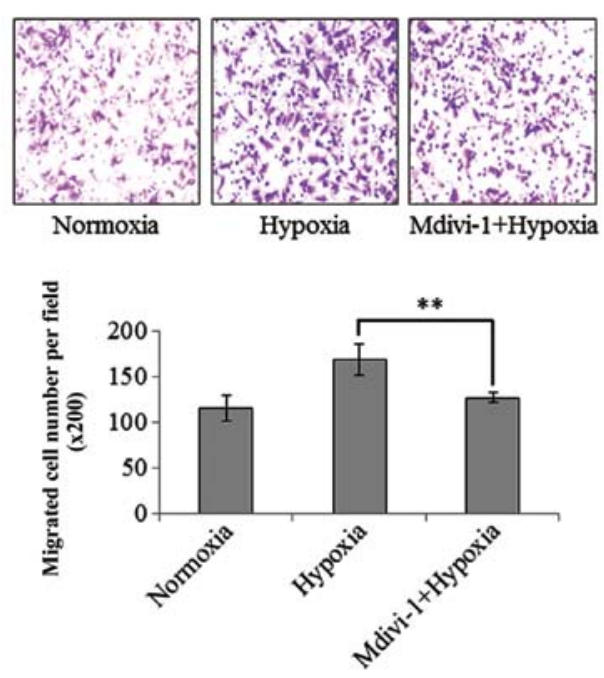

Figure 3. Effect of Mdivi-1 on hypoxia-induced migration of U251 cells (A) Cells were pre-treated with $5 \mu \mathrm{M}$ of Mdivi-1 or vehicle. One hour later, cells were incubated with $100 \mu \mathrm{M}$ of $\mathrm{CoCl}_{2}$ or the same volume of PBS (Cont). Cell migration was examined by scratch assay at indicated time points. Scale bar, $100 \mu \mathrm{m}$. (B) After pre-treatment with $5 \mu \mathrm{M}$ of Mdivi-1 or vehicle, cells were exposed to normoxia or hypoxia for $6 \mathrm{~h}$. Cell migration was evaluated by Transwell assay. Migrated cells were visualized by microscopy and counted in 10 fields for each treatment. ${ }^{* *} \mathrm{p}<0.01$.

migration of cancer cells. Firstly, the migration of U251 cells under normoxia and hypoxia was evaluated by scratch and Transwell assays. In scratch assay, $\mathrm{CoCl}_{2}$ treatment was used to mimic the hypoxia. Consistent with previous studies (5), both scratch and Transwell assays indicated that hypoxia significantly enhanced the migration of U251, shown in Fig. 3. To investigate the role of Drp1 in hypoxia-induced migration of U251 cells, the effect of Mdivi-1 was evaluated. Mdivi-1 is the first selective inhibitor of Drp1, which inhibits Drp1 self-assembly and attenuates mitochondrial fission (25). Mdivi-1 has been applied to prevent mitochondrial fission in a number of disease models $(26,27)$. Results showed pretreatment with $5 \mu \mathrm{M}$ of Mdivi-1 significantly attenuated hypoxia-induced migration of U251 cells, shown in Fig. 3. However, recent studies suggest that Mdivi-1 could potentially have off-target effects on preventing mitochondrial outer membrane permeabilization (28). Therefore, further investigations are required to directly demonstrate the involvement of Drp1 in hypoxia-induced migration of U251 cells. 
A
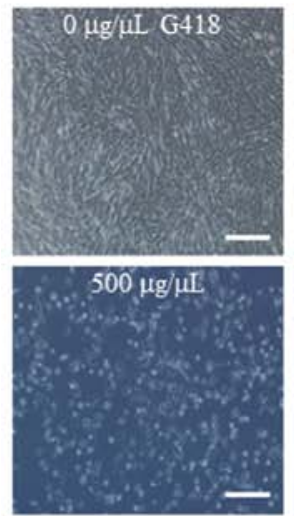

B

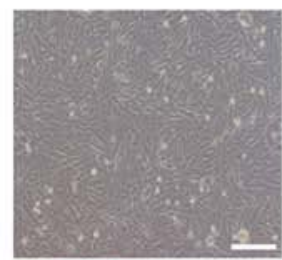

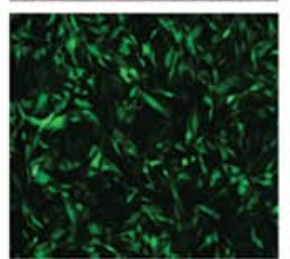

GFP
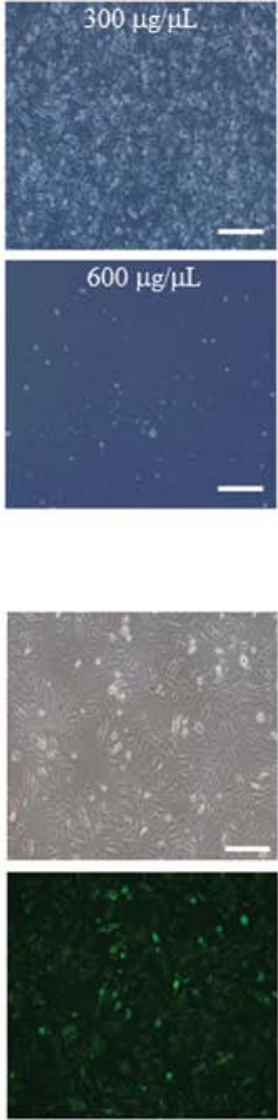

GFP-Drp1
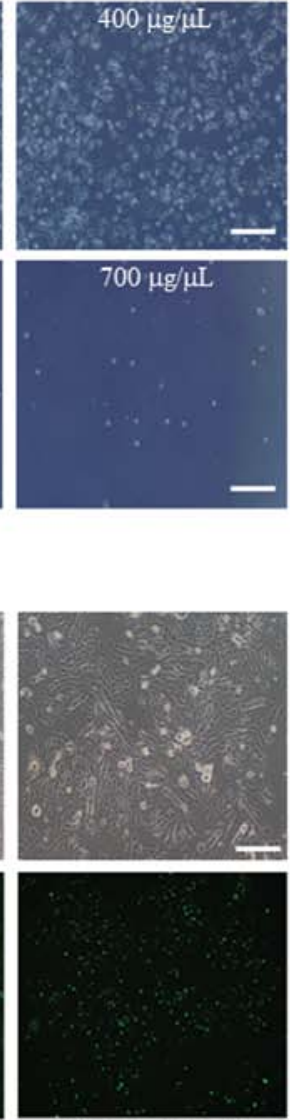

GFP-Drp1-K38A
C

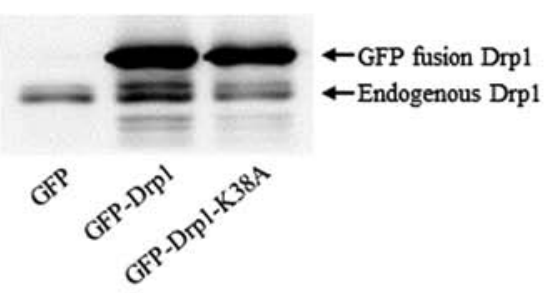

Figure 4. Establishment of U251 stable cell lines expressing GFP, GFP-Drp1 or GFP-Drp1 K38A. (A) Determination of the minimum lethal concentration of G418 to U251 cells. U251 cells were cultured in $0-1,100 \mu \mathrm{g} / \mathrm{ml}$ of G418 for 12 days. The images under bright-field microscope showed the survival state of cells growing at different concentrations of G418. The minimum concentration of G418 induced complete cell death was decided as the minimum lethal concentration of G418 to U251 cells. Scale bar, $100 \mu \mathrm{m}$. (B) Confirmation of three U251 cell lines under fluorescence microscope. The upper panels are images of three cell lines from bright-field, and the lower panels are from fluorescence microscope. Scale bar, $100 \mu \mathrm{m}$. (C) Expression of endogenous and exogenous Drp1 in three U251 cell lines. Cell lysate of three cell lines was subjected to $8 \%$ SDS-PAGE and immunoblotted with Drp1 antibody. The upper bands were GFP fusion Drp1 in GFP-Drp1 and GFP-Drp1 K38A cell lines. The lower bands were the endogenous Drp1. Drp1, dynamin-related protein 1.

Establishment of the U251 cell lines stably expressing GFP, GFP-Drpl or GFP-Drp1-K38A. The dominant negative mutation of K38A in Drp1 has been well identified, and Drp1-K38A is a frequently used tool to investigate the direct role of Drp1 in cellular activities $(29,30)$. To explore the direct involvement of Drp1 in hypoxia-induced migration, three U251 cell lines
A

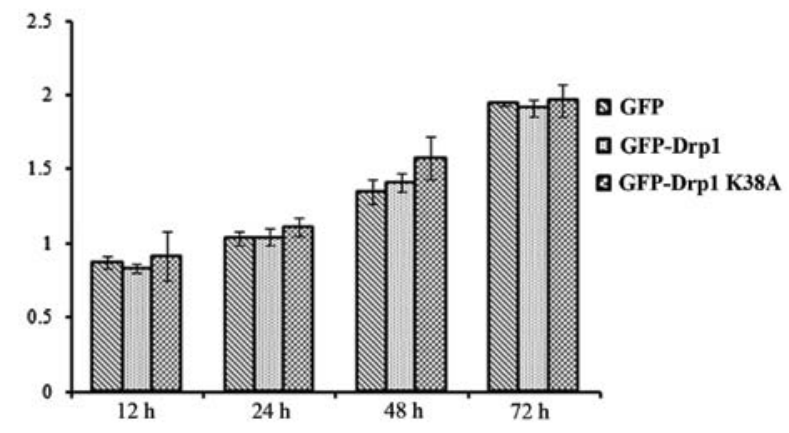

B

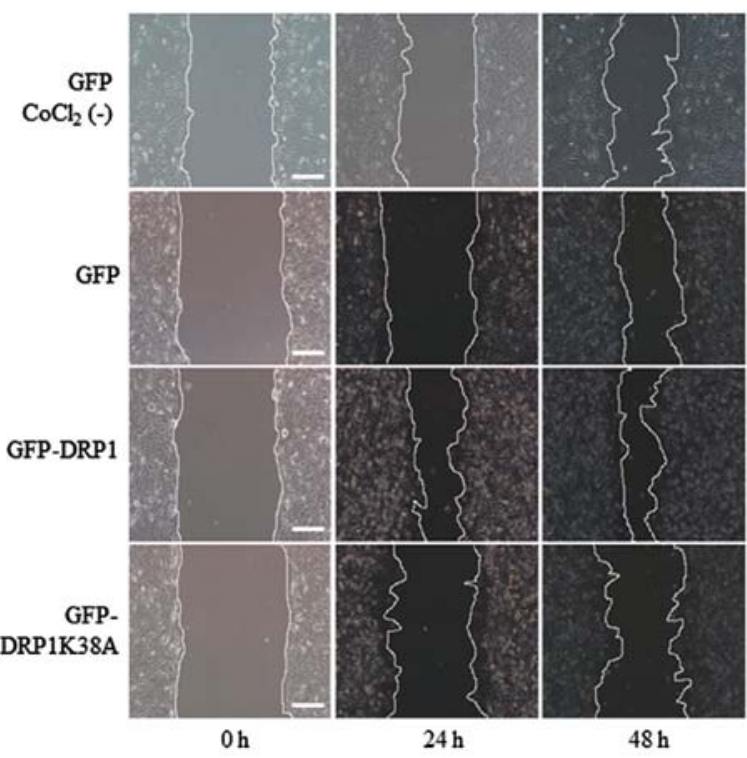

Figure 5. Exogenously expressed Drp1 and Drp1 K38A on cell proliferation and hypoxia-induced migration. (A) The proliferation of three U251 stable cell lines expressing GFP, GFP-Drp1 or GFP-Drp1 K38A was assessed by the MTT assay at indicated time points. The values for GFP-Drp1 and GFP-Drp1 K38A cell lines were normalized to that of the GFP cell line. (B) Migration of three cell lines after $\mathrm{CoCl}_{2}$ treatment. Three $\mathrm{U} 251$ cell lines were treated with $100 \mu \mathrm{M}$ of $\mathrm{CoCl}_{2}$ or the same volume PBS $\left[\mathrm{CoCl}_{2}(-)\right]$, and the migration of $\mathrm{U} 251$ cells was evaluated by scratch assay at indicated time points. Scale bar, $100 \mu \mathrm{m}$. Drp1, dynamin-related protein 1 .

stably expressing GFP, GFP-Drp1 or GFP-Drp1-K38A were established as previously described (22). Firstly, the minimum lethal concentration of G418 to U251 cells was determined as $600 \mu \mathrm{g} / \mu \mathrm{l}$, as shown in Fig. 4A. Thus, $700 \mu \mathrm{g} / \mu \mathrm{l}$ of G418 was used for selection of U251 stable cell lines. The stable cell colonies were confirmed by fluorescence microscopy and western blot assay. As shown in Fig. 4B, GFP fluorescence was clearly visualized in three cell lines. Consistent with a previous report (29), GFP-Drp1 K38A accumulated into large aggregates and punctate foci, while GFP and GFP-Drp1 were mostly diffused in cytoplasm. In addition, the results of western blotting showed the endogenously and exogenously expressed Drp1 in three stable cell lines. As shown in Fig. 4C, several bands were detected in GFP-Drp1 and GFP-Drp1 K38A cell lines. The upper band was GFP fused Drp1 or Drp1 K38A, and the lower bands was endogenous Drpl. The results of fluorescence microscopy and western blotting indicated that three U251 cell 
$\mathbf{A}$

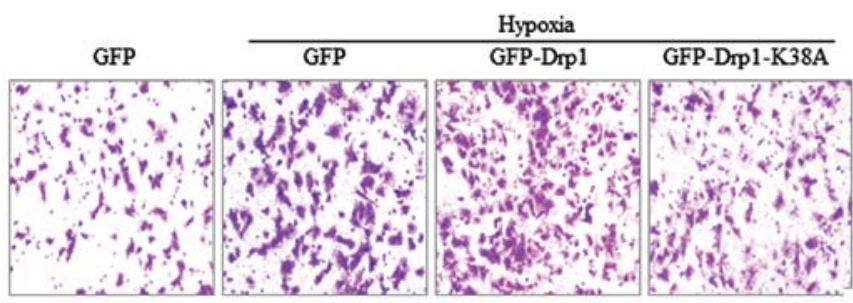

$\mathbf{B}$

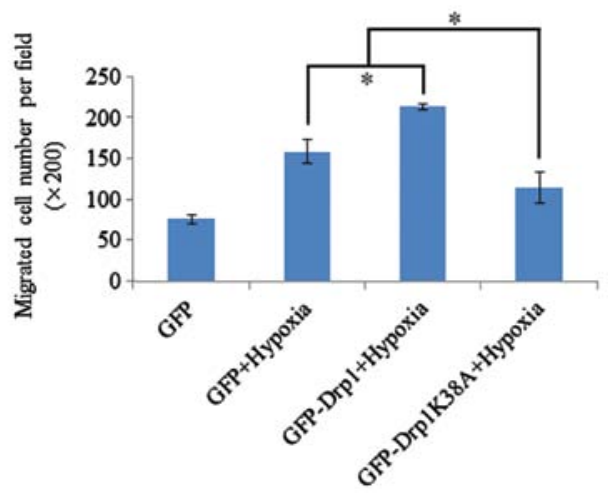

Figure 6. Quantification of migration of three U251 stable cell lines under hypoxia. (A) Migratory activity was evaluated by Transwell assay. Three stable cell lines exposed to normoxia or hypoxia for $6 \mathrm{~h}$. Migrated cells were stained with crystal violet and visualized by microscopy. (B) Quantification of migrated cells. Migrated cells in three stable cell lines were counted in 10 fields for each treatment. " $\mathrm{p}<0.01$.

lines stably expressing GFP, GFP-Drp1 and GFP-Drp1 K38A were successfully established.

Effect of exogenously expressed Drpl and Drpl-K38A on hypoxia-induced migration of U251 cells. The result of the scratch assay may be influenced by cell proliferation. Thus, we examined the effect of exogenously expressed Drp1 and Drp1-K38A on cell proliferation by MTT assay, prior to evaluation of the migration of three cell lines. As shown in Fig. 5A, MTT assay indicated that exogenously expressed Drp1 or Drp1 K38A had no significant effect on cell proliferation. To elucidate the role of Drp1 in hypoxia-induced migration, the migratory activity of three stable cell lines expressing GFP, GFP-Drp1 or GFP-Drp1 K38A was evaluated after $\mathrm{CoCl}_{2}$ or hypoxia treatment. Scratch and Transwell assay showed that exogenously expressed Drp1-K38A efficiently attenuated the migration of U251 cells, while exogenously expressed Drp1 enhanced the migratory activity of U251 cells under hypoxia (Figs. 5B and 6). Collectively, these results demonstrate the involvement of Drp1 in hypoxia-induced migration of human glioblastoma U251 cells.

\section{Discussion}

Cancer cells are characterized by their uncontrolled high proliferation. The rapid proliferation always results in a hypoxic microenvironment in the central region of solid tumors. It has been well documented that the hypoxic microenvironment in tumors plays an important role in migration and invasion of cancer cells $(4,5)$. HIF-1 $\alpha$, a hypoxia-inducible factor, is the vital transcription factor activated by hypoxia, which promotes the adaptation of cancer cells to the hypoxic microenvironment through upregulating gene expression related to metabolism, cellular mobility and angiogenesis, such as GLUT1, MMPs and $\operatorname{VEGF}(4,8,9)$. On the other hand, mitochondrial functions are crucial for cancer survival, proliferation and metastasis through regulation of ATP production, $\mathrm{Ca}^{2+}$ homeostasis, cell cycle and apoptosis (11-13). Mitochondria are also dynamic organelles in cells with frequent fission and fusion processes (14). Recent studies have provided insight into the involvement of Drp1dependent mitochondrial fission in cancer cells (15-20). Drp1 is a highly conserved dynamin-related GTPase in mammalian cells, and its GTPase activity may be regulated by some post-transcriptional modification, such as phosphorylation, S-nitrosylation, ubiquitination and SUMOylation (31-35). The high expression of Drp1 was recently found in several cancers, such as neuroblastoma, breast and lung cancer. Inhibition of Drp1 has been found to efficiently reduce cancer cell growth and enhance spontaneous apoptosis in cancer (27).

In the present study, we explored whether Drp1 is involved in the hypoxia-induced migration of human glioblastoma U251 cells. It was found that hypoxia significantly promoted the transcription and expression of Drp1, and stimulated Drp1-dependent mitochondrial fission (Figs. 1 and 2). HIF-1 $\alpha$ inhibitor echinomycin efficiently blocked hypoxia-induced expression of Drp1 (Fig. 1B). Consistent with previous studies (5), hypoxia significantly enhanced the migratory activity of U251 cells (Fig. 3). To examine the possible role of Drp1 in hypoxia-induced migration, pharmacological experiments were performed using Mdivi-1, a Drp1 inhibitor, which attenuates Drp1 self-assembly. Mdivi-1 (5 $\mu \mathrm{M})$ efficiently attenuated hypoxia-induced mitochondrial fission and migration of U251 cells (Figs. 2B and 3). These results suggest that hypoxia may enhance migration of U251 cells through upregulation of Drp1 via HIF-1 $\alpha$. However, the Drp1 inhibitor Mdivi-1 could have some off-target effects, such as Drp1-independent effect on mitochondrial outer membrane permeabilization (MOMP) (28). Changes in MOMP and other mitochondrial dysfunctions may regulate the migration of cancer cells via reactive oxygen species $(37,38)$. To elucidate the direct role of Drp1 in hypoxia-induced migration, three U251 stable cell lines expressing GFP, GFP-Drp1 or GFP-Drp1 K38A were established. Compared with the GFP cell line, stable expression of Drp1 or dominant negative Drp1 K38A respectively enhanced or attenuated the migration of U251 cells under hypoxia (Figs. 5B and 6). Therefore, the present study demonstrated the involvement of Drp1 in hypoxia-induced migration of human glioblastoma U251 cells. These results are consistent with a recent study, which demonstrated the involvement of Drp1-dependent mitochondrial fission in migration of breast cancer cells through modifying lamellipodial formation (17). However, a major challenge for further studies will be to identify the molecular pathway through which Drp1 regulates hypoxia-induced migration of U251 cells.

In contrast, another recent study reported that hypoxia induced upregulation of mitochondrial fusion protein Mfn1 in some types of cancer cells (39). The upregulation of Mfn1 and two Bcl-2 family members, BNIP3 and BNIP3L, participates in the formation of enlarged mitochondria under 
hypoxia. Hypoxic enlarged mitochondria efficiently enhance the resistance of cancer cells to chemotherapy. However, this phenomenon was only observed in some specific types of cancer cell lines, such as LS174, A549, HeLa and 786-O cells, but not in PC3 (prostate cancer), MCF7 (breast cancer), CAL33, ORL3 (head and neck) and SkMel (melanoma) cells. In our present study, hypoxia upregulated mitochondrial fission protein Drp1 and enhanced the migration of glioblastoma U251 cells. The different effect of hypoxia on the expression of mitochondria related proteins and the activity of cancer cells is possibly due to the different cancer cell lines. It is also worth investigating the underlying mechanism of the effect of hypoxia on different types of cancer cells.

In conclusion, our data show for the first time that Drp1 is involved in hypoxia-induced migration of glioblastma U251 cells. Hypoxia promotes the migratory activity of U251 cells through the upregulation of Drp1 expression. Inhibition of Drp1 activity by Mdivi-1 or expression of dominant negative Drp1 K38A efficiently attenuates hypoxia-induced migration of U251 cells. Glioblastomas are highly aggressive brain tumors; prevention of migration and invasion is critical to improve therapeutic effect and prognosis. Thus, our data suggest that mitochondrial fission protein Drp1 may be a potential target for the prevention and therapy of glioblastoma in the future.

\section{Acknowledgements}

This study was supported by the National Natural Science Foundation of China (31360241), the Postgraduate Student Foundation for New Teacher from the Ministry of Education of China (20123601120001), and the Foundation from the Department of Education of Jiangxi Province (GJJ13162).

\section{References}

1. Behin A, Hoang-Xuan K, Carpentier AF and Delattre JY: Primary brain tumours in adults. Lancet 361: 323-331, 2003.

2. Claes A, Idema AJ and Wesseling P: Diffuse glioma growth: a guerilla war. Acta Neuropathol 114: 443-458, 2007.

3. Anton K, Baehring JM and Mayer T: Glioblastoma multiforme: overview of current treatment and future perspectives. Hematol Oncol Clin North Am 26: 825-853, 2012.

4. Chan DA and Giaccia AJ: Hypoxia, gene expression, and metastasis. Cancer Metastasis Rev 26: 333-339, 2007.

5. Zhang Y, Liu Q, Wang F, Ling EA, Liu S, Wang L, Yang Y, Yao L, Chen X, Wang F, Shi W, Gao M and Hao A: Melatonin antagonizes hypoxia-mediated glioblastoma cell migration and invasion via inhibition of HIF-1 $\alpha$. J Pineal Res 55: 121-130, 2013.

6. Chan DA, Sutphin PD, Yen SE and Giaccia AJ: Coordinate regulation of the oxygen-dependent degradation domains of hypoxia-inducible factor $1 \alpha$. Mol Cell Biol 25: 6415-6426, 2005

7. Chan DA, Sutphin PD, Denko NC and Giaccia AJ: Role of prolyl hydroxylation in oncogenically stabilized hypoxia-inducible factor-1 $\alpha$. J Biol Chem 277: 40112-40117, 2002.

8. Krishnamachary B, Berg-Dixon S, Kelly B, Agani F, Feldser D, Ferreira G, Iyer N, LaRusch J, Pak B, Taghavi P and Semenza GL: Regulation of colon carcinoma cell invasion by hypoxia-inducible factor 1. Cancer Res 63: 1138-1143, 2003.

9. Muñoz-Nájar UM, Neurath KM, Vumbaca F and Claffey KP: Hypoxia stimulates breast carcinoma cell invasion through MT1-MMP and MMP-2 activation. Oncogene 25: 2379-2392, 2006.

10. Zagzag D, Zhong H, Scalzitti JM, Laughner E, Simons JW and Semenza GL: Expression of hypoxia-inducible factor $1 \alpha$ in brain tumors: association with angiogenesis, invasion, and progression. Cancer 88: 2606-2618, 2000

11. Oakes SA and Korsmeyer SJ: Untangling the web: mitochondrial fission and apoptosis. Dev Cell 7: 460-462, 2004.
12. Szabadkai G, Simoni AM, Chami M, Wieckowski MR, Youle RJ and Rizzuto R: Drp-1-dependent division of the mitochondrial network blocks intraorganellar $\mathrm{Ca}^{2+}$ waves and protects against $\mathrm{Ca}^{2+}$-mediated apoptosis. Mol Cell 16: 59-68, 2004.

13. Shaw JM and Nunnari J: Mitochondrial dynamics and division in budding yeast. Trends Cell Biol 12: 178-184, 2002.

14. Chan DC: Mitochondrial fusion and fission in mammals. Annu Rev Cell Dev Biol 22: 79-99, 2006.

15. Grandemange S, Herzig S and Martinou JC: Mitochondrial dynamics and cancer. Semin Cancer Biol 19: 50-56, 2009.

16. Rehman J, Zhang HJ, Toth PT, Zhang Y, Marsboom G, Hong Z, Salgia R, Husain AN, Wietholt C and Archer SL: Inhibition of mitochondrial fission prevents cell cycle progression in lung cancer. FASEB J 26: 2175-2186, 2012.

17. Zhao J, Zhang J, Yu M, Xie Y, Huang Y, Wolff DW, Abel PW and Tu Y: Mitochondrial dynamics regulates migration and invasion of breast cancer cells. Oncogene 32: 4814-4824, 2013.

18. Hagenbuchner J, Kuznetsov AV, Obexer P and Ausserlechner MJ: BIRC5/Survivin enhances aerobic glycolysis and drug resistance by altered regulation of the mitochondrial fusion/fission machinery. Oncogene 32: 4748-4757, 2013.

19. Qian W, Choi S, Gibson GA, Watkins SC, Bakkenist CJ and Van Houten B: Mitochondrial hyperfusion induced by loss of the fission protein Drp1 causes ATM-dependent G2/M arrest and aneuploidy through DNA replication stress. J Cell Sci 125: 5745-5757, 2012.

20. Inoue-Yamauchi A and Oda H: Depletion of mitochondrial fission factor DRP1 causes increased apoptosis in human colon cancer cells. Biochem Biophys Res Commun 421: 81-85, 2012.

21. Parone PA, Da Cruz S, Tondera D, Mattenberger Y, James DI, Maechler P, Barja F and Martinou JC: Preventing mitochondrial fission impairs mitochondrial function and leads to loss of mitochondrial DNA. PLoS One 3: e3257, 2008.

22. $\mathrm{Hu} \mathrm{D}, \mathrm{Wu} \mathrm{J}, \mathrm{Xu} \mathrm{L}$, Zhang R and Chen L: A method for the establishment of a cell line with stable expression of the GFP-LC3 reporter protein. Mol Med Rep 6: 783-786, 2012.

23. Marsboom G, Toth PT, Ryan JJ, et al: Dynamin-related protein 1-mediated mitochondrial mitotic fission permits hyperproliferation of vascular smooth muscle cells and offers a novel therapeutic target in pulmonary hypertension. Circ Res 110: 1484-1497, 2012.

24. Wang GL and Semenza GL: Desferrioxamine induces erythropoietin gene expression and hypoxia-inducible factor 1 DNA-binding activity: implications for models of hypoxia signal transduction. Blood 82: 3610-3615, 1993.

25. Cassidy-Stone A, Chipuk JE, Ingerman E, Song C, Yoo C, Kuwana T, Kurth MJ, Shaw JT, Hinshaw JE, Green DR and Nunnari J: Chemical inhibition of the mitochondrial division dynamin reveals its role in Bax/Bak-dependent mitochondrial outer membrane permeabilization. Dev Cell 14: 193-204, 2008.

26. Lackner LL and Nunnari J: Small molecule inhibitors of mitochondrial division: tools that translate basic biological research into medicine. Chem Biol 17: 578-583, 2010.

27. Qian W, Wang J and Van Houten B: The role of dynamin-related protein 1 in cancer growth: a promising therapeutic target? Expert Opin Ther Targets 17: 997-1001, 2013.

28. Kushnareva Y, Andreyev AY, Kuwana T and Newmeyer DD: Bax activation initiates the assembly of a multimeric catalyst that facilitates Bax pore formation in mitochondrial outer membranes. PLoS Biol 10: e1001394, 2012.

29. Pitts KR, Yoon Y, Krueger EW and McNiven MA: The dynaminlike protein DLP1 is essential for normal distribution and morphology of the endoplasmic reticulum and mitochondria in mammalian cells. Mol Biol Cell 10: 4403-4417, 1999.

30. Ong SB, Subrayan S, Lim SY, Yellon DM, Davidson SM and Hausenloy DJ: Inhibiting mitochondrial fission protects the heart against ischemia/reperfusion injury. Circulation 121: 2012-2022, 2010.

31. Taguchi N, Ishihara N, Jofuku A, Oka T and Mihara K: Mitotic phosphorylation of dynamin-related GTPase Drp1 participates in mitochondrial fission. J Biol Chem 282: 11521-11529, 2007.

32. Han XJ, Lu YF, Li SA, Kaitsuka T, Sato Y, Tomizawa K, Nairn AC, Takei K, Matsui $\mathrm{H}$ and Matsushita M: CaM kinase I $\alpha$-induced phosphorylation of Drp1 regulates mitochondrial morphology. J Cell Biol 182: 573-585, 2008.

33. Cho DH, Nakamura T, Fang J, Cieplak P, Godzik A, Gu Z and Lipton SA: S-nitrosylation of Drpl mediates $\beta$-amyloid-related mitochondrial fission and neuronal injury. Science 324: 102-105, 2009. 
34. Wang H, Song P, Du L, Tian W, Yue W, Liu M, Li D, Wang B, Zhu Y, Cao C, Zhou J and Chen Q: Parkin ubiquitinates Drpl for proteasome-dependent degradation: implication of dysregulated mitochondrial dynamics in Parkinson disease. J Biol Chem 286: 11649-11658, 2011.

35. Harder Z, Zunino R and McBride H: Sumol conjugates mitochondrial substrates and participates in mitochondrial fission. Curr Biol 14: 340-345, 2004.

36. Piret JP, Mottet D, Raes M and Michiels C: $\mathrm{CoCl}_{2}$, a chemical inducer of hypoxia-inducible factor-1, and hypoxia reduce apoptotic cell death in hepatoma cell line HepG2. Ann NY Acad Sci 973: 443-447, 2002.
37. Ma J, Zhang Q, Chen S, Fang B, Yang Q, Chen C, Miele L, Sarkar FH, Xia J and Wang Z: Mitochondrial dysfunction promotes breast cancer cell migration and invasion through HIF1 $\alpha$ accumulation via increased production of reactive oxygen species. PLoS One 8: e69485, 2013.

38. Tochhawng L, Deng S, Pervaiz S and Yap CT: Redox regulation of cancer cell migration and invasion. Mitochondrion 13: 246-253, 2013

39. Chiche J, Rouleau M, Gounon P, et al: Hypoxic enlarged mitochondria protect cancer cells from apoptotic stimuli. J Cell Physiol 222: 648-657, 2010. 